\title{
Stress and Burnout in Postgraduates in a Medical College
}

\author{
Authors \\ Dr Galina Lisa D'Souza*, Dr Safeekh A.T., Dr Siddharth Shetty \\ Father Muller Medical college Hospital, Mangalore \\ *Corresponding Author \\ Dr Galina Lisa D'Souza \\ Junior Resident, Father Muller Medical College Hospital, Mangalore
}

\begin{abstract}
Introduction: Medicine is associated not only with a great degree of both personal and professional satisfaction, but also a high level of occupational stress and burnout. Resident doctors/trainees and faculty members, are prone to developing mental health problems such as depression, anxiety, and substance abuse

Objectives:

1. To understand the domains most affected with burnout and stress

2. To compare stress levels among postgraduates of various years and specialties

Methods: Postgraduates (MD/MS/Diploma) from batches 2016,2017 and 2018 were given the self administered questionnaires-cohen's perceived stress scale and copenhagen burnout inventory -personal, work and client burnout after institutional ethical approval along with dean's approval and informed consent Results: Highest perceived stress was seen in general surgery residents (mean score of 22) While the least was seen in Ophthalmology residents (score of 14)

Maximum personal burnout was seen in General surgery residents (ie.73.9\%) followed by OBG and paediatrics whereas work burnout was again highest in general surgery(65.1\%) followed by orthopaedics and paediatrics .client burnout, $O B G(80.33)$ was the highest followed by general surgery (51.1\%) Perceived stress was higher among the juniors when compared to the seniors.

Conclusion: Stress and Burnout was more in surgical than medical fields. Stress is one of the major growing mental problems among highly educated health professional and it should not be ignored as it can cause many other health issues. Institutions and national policy makers should take into account this unsettling issue of burnout in doctors -especially residents and implement preventive strategies

Keywords: Stress, Burnout, Postgraduates.
\end{abstract}

\section{Introduction}

Studies across the world suggest that health-care professionals, especially resident doctors/trainees and faculty members, are prone to developing mental health problems such as depression, anxiety, and substance abuse. It is Often attributed to the requirement of dedication and precision on the part of the health-care providers ${ }^{1}$. The need for urgent care and long hours at work further add to the stress. Long-standing stress in medical professionals 
can often lead to burnout ${ }^{2}$. Internship and residency are difficult times with novice practitioners facing new challenges and stressors ${ }^{3}$. Sources of such stress in resident training can be divided into three components: situational (workload, sleep deprivation, and poor learning environment); personal (family, isolation, and financial); professional (overwhelming patient responsibility and information).Burnout is a syndrome that encompasses three dimensions: emotional exhaustion, depersonalisation and reduced personal accomplishment. It can be a jeopardizing condition for a medical graduate during the years of acquiring proficiency $^{4}$

\section{Objectives of the Study}

- To understand the domains most affected with burnout and stress

- To compare stress levels among postgraduates of various years and specialties

\section{Methodology}

248 Postgraduates from 2016, 2017 and 2018 were given the self administered questionnaires Cohen's Perceived stress scale and copenhagen burnout inventory (3 domains - personal, work and client) after informed consent, permission from the dean and ethical clearance. The Inclusion criteria Medical Postgraduates from clinical, paraclinical and preclinical branches and Exclusion criteria: Undergraduates and allied health postgraduates those with a prior/current history of psychiatric illness were excluded

\section{Scales}

1. Perceived stress scale (PSS) is the most widely used 10 item instrument for measuring the perception of stress and is designed to measure the degree to which situations in one's life are appraised as stressful.The tool, while originallydeveloped in 1983, remains a popular choice for helping us understand how different situations affect feelings and perceived stress. The questions in this scale ask about feelings and thoughts during the last month. ${ }^{5}$

2. Copenhagen Burnout Inventory (CBI) is a reliable and validated 19-item questionnaire. It focuses on exhaustion and its attribution by the person. The CBI has scales on personal burnout, work-related burnout and client-related burnout.All items have five response categories in a Likert scale.CBI was chosen over Maslach Burnout Inventory (MBI) as it was found to have similar psychometric properties and was available for free ${ }^{6}$.

\section{Statistical Analysis}

Data was analysed by frequency distribution, mean, median and standard deviation using SPSS Version 23

\section{Results}

The response rate was $22 \%$ that is around 56 postgraduates responded. Responses were received from ENT, dermatology, anaesthsiology, Medicine, General Surgery, Microbiology, OBG, Ophthalmology, Orthopedics, Radiology, Paediatrics, Pathology, Pharmacology and Psychiatry.

No responses were received from radiotherapy. There were no postgraduates in the preclinical departments

Highest perceived stress was seen in general surgery residents (mean score of 22) While the least was seen in Ophthalmology residents (score of 14) (Fig 1).Perceived stress was higher in juniors when compared to the seniors (Fig 2) 


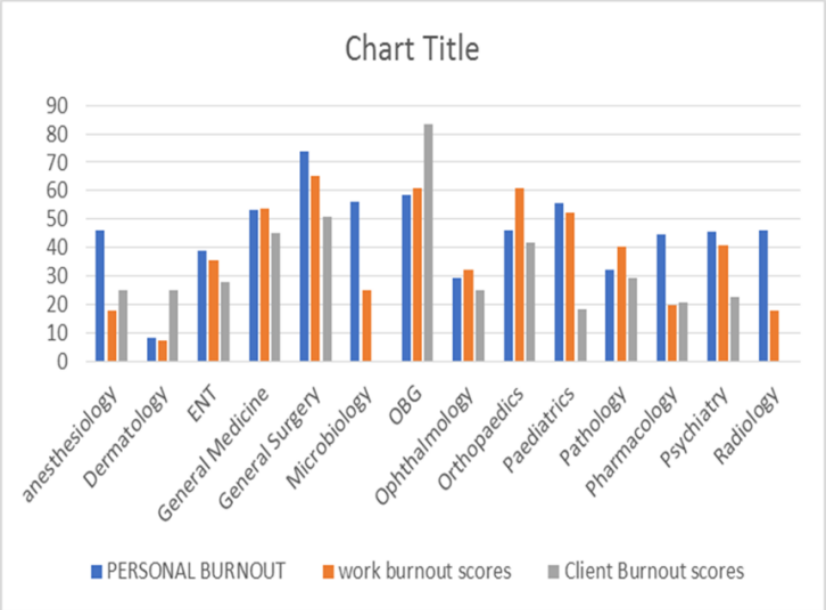

Fig 1

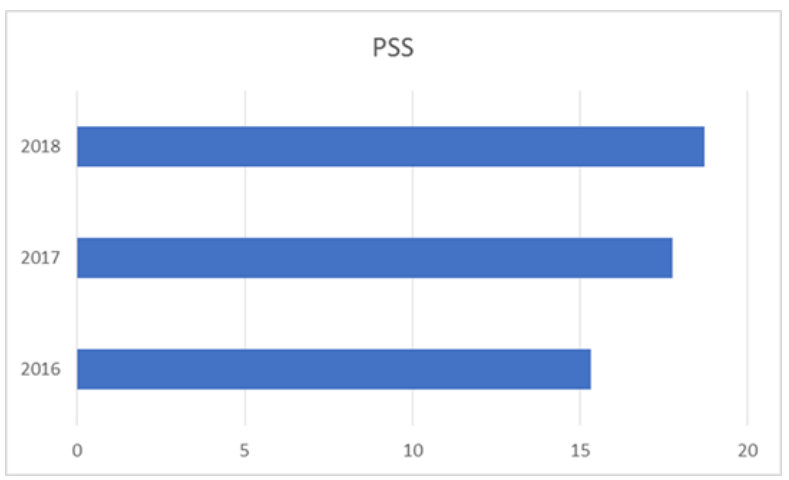

Fig 2

Maximum personal burnout was seen in General surgery residents (ie.73.9\%) followed by OBG and paediatrics. work burnout was again highest in general surgery $(65.1 \%)$ followed by orthopaedics and paediatrics .client burnout -OBG (80.33) was the highest followed by general surgery $(51.1 \%)$ (Fig 3)

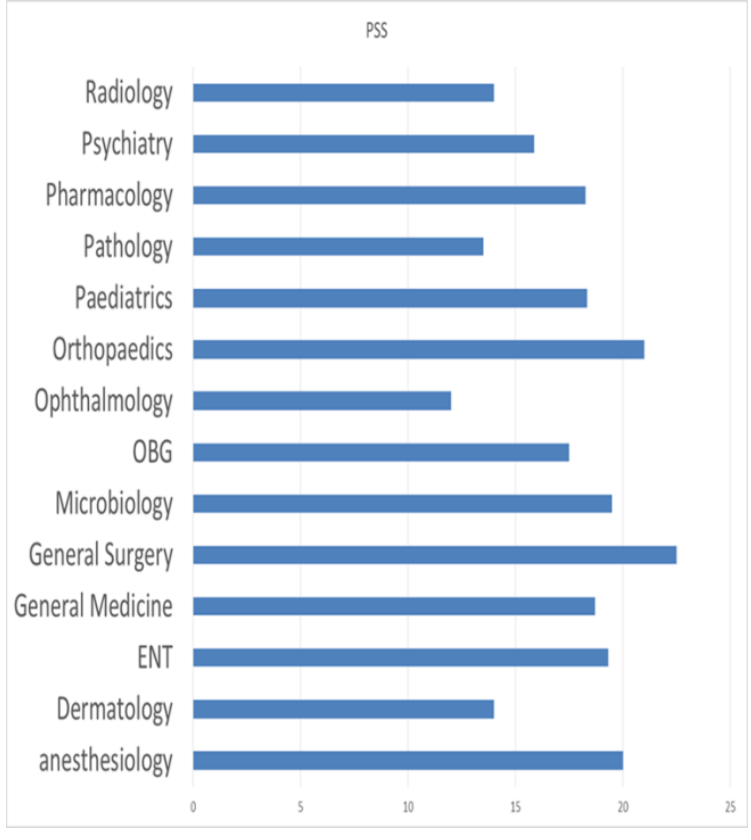

Fig 3

\section{Sociodemographic data}

$67.9 \%$ were females, rest were males $.87 .5 \%$ had done their undergraduation from a private medical college.Only $19.6 \%$ were married, mean age of the postgraduate was about 27 years $.69 .6 \%$ of the Postgraduates had moderate stress levels $.46 .4 \%$ had personal burnout (>50score), $42.9 \%$ had work burnout and $21.4 \%$ had client burnout .46 .4 responses were from 2017 batch followed by 2018(32.1\%). $73.2 \%$ were MD candidates followed by MS candidates (16.1\%)

The mean scores of burnout out are given Fig 4

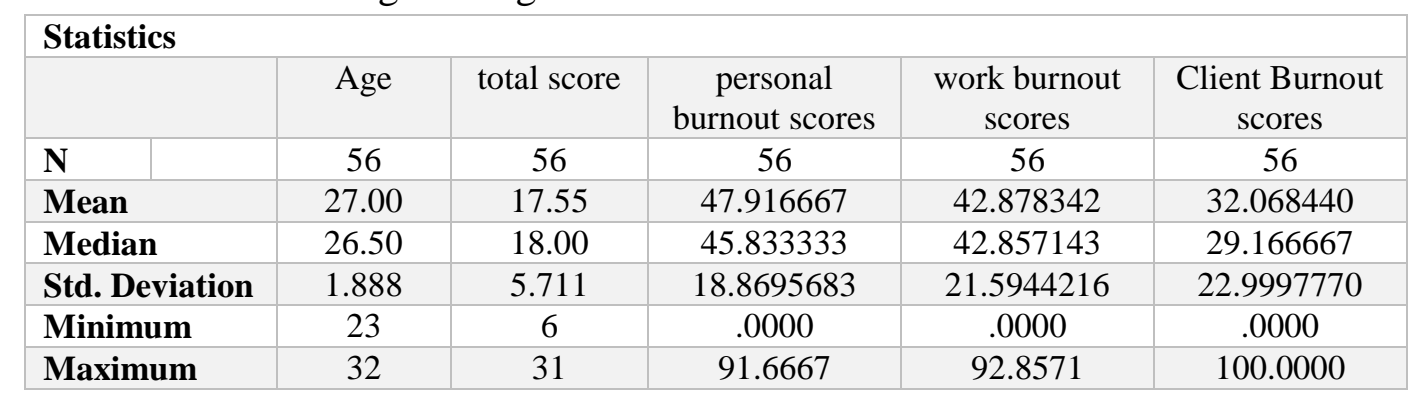




\section{Discussion}

Our Study Was Comparable to a study by B ratnakaran et al that is Non Medical/Non Surgical residents had the least prevalence of burnout in all three dimensions, whereas surgical specialty residents had the highest personal burnout (57.92 $\%)^{7}$ The mean PSS was around 17.5 which was lower than a Saudi study done by Fahad et al which reported a mean score of $22^{8}$ The perceived stress among residents in the current study appeared to be comparable to or slightly lower than the perceived stress reported among residents in other parts of the world. Our study four -fifth had a moderate to low stress level as compared to a study which had fourfifth of the participants report moderate-to-high stress levels. Study done by Datar M et al used the PSS, assessment of the domains of the stressors revealed $88 \%$ reported stress about their academic performance, $83 \%$ residents had stress due to responsibilities at workplace, $77.5 \%$ were stressed about their future career, $66 \%$ reported stress due to interpersonal problems at work, $45.5 \%$ had stress of personal relationships, $39.5 \%$ had stress related to financial issues, and $36.5 \%$ reported stress due to family problems. ${ }^{9}$ Yusoff and Rahim et al also stated that the major stressors in postgraduate medical training were related to academic and performance pressure ${ }^{10}$. No studies exclusively on postgraduate burnout using CBI were found

\section{Conclusion}

Stress and Burnout was more in surgical than medical fields. One of the major growing mental problems among highly educated health professional and it should not be ignored as it can cause many other health issues. Institutions and national policy makers should take into account this unsettling issue of burnout in doctors -especially residents and implement preventive strategies

\section{Limitations}

The response rate to the survey was only $22 \%$ as many feared repercussions of their identity possibly being disclosed to their respective departments. Lack of interest in participating in the study

Study has not taken into account factors such as patient load and average working hours with respect to different departments

\section{References}

1. Grover S, Sahoo S, Bhalla A, Avasthi A. Psychological problems and burnout among medical professionals of a tertiary care hospital of North India: A cross-sectional study. Indian J Psychiatry 2018;60(2):175.

2. Bhatia M, Saha R. Burnout in medical residents: A growing concern. J Postgrad Med [Internet]. 2018;64(3):136. Available from:

http://www.jpgmonline.com/text.asp?2018/6 4/3/136/236376

3. Gunasingam N, Burns K, Edwards J, Dinh M, Walton M. Reducing stress and burnout in junior doctors: The impact of debriefing sessions. Postgrad Med J. 2015;91(1074):182-7.

4. Goldhagen B, Kingsolver K, Stinnett S, Rosdahl J. Stress and burnout in residents: Impact of mindfulness-based resilience training. Adv Med EducPract.2015;6:525327

5. Cohen S, Kamarck T, Mermelstein R. Perceived stress scale. Measuring stress: A guide for health and social scientists. 1994;10.

6. Kristensen TS, Borritz M, Villadsen E, Christensen KB. The Copenhagen Burnout Inventory: A new tool for the assessment of burnout. Work Stress. 2005;19(3):192-207.

7. Ratnakaran B, Prabhakaran A, Karunakaran V. Prevalence of burnout and its correlates among residents in a tertiary medical center 
in Kerala, India: A cross-sectional study. J Postgrad Med. 2016;62(3):157-161. doi:10.4103/0022-3859.184274

8. Alosaimi, Fahad \& Kazim, Sana \& Almufleh, Auroabah \& Aladwani, Bandar \& Alsubaie, Abdullah. (2015). Prevalence of stress and its determinants among residents in Saudi Arabia. Saudi medical journal. 36. 605-612. 10.15537/smj.2015.5.10814.

9. Datar MC, Shetty JV, Naphade NM. Stress and coping styles in postgraduate medical students: A medical college-based study. Indian Journal of Social Psychiatry. 2017 Oct 1;33(4):370.

10. Yusoff MS, Rahim AF. Prevalence and sources of stress among postgraduate medical trainees: initial findings. ASEAN Journal of Psychiatry. 2010 Jul;11(2):180-9. 